Recepción: 24/04/2014

Evaluación: 20/05/2014

Aprobación: 27/08/2014

Artículo de Reflexión

\title{
ESTADO DEL ARTE DEL RENDIMIENTO ACADÉMICO EN LA EDUCACIÓN MEDIA
}

\author{
Oscar Hernán Cerquera Losada \\ Universidad Surcolombiana \\ Grupo de investigación IGUAQUE \\ os-celo@hotmail.com
}

\begin{abstract}
RESUMEN
Este documento muestra el Estado de arte de los determinantes del rendimiento académico en la educación media, teniendo en cuenta las principales investigaciones realizadas, tanto a nivel nacional como internacional, acerca de los factores que influyen en el logro escolar de los estudiantes. Con este trabajo, se busca establecer las principales variables, tanto en Colombia como en algunos lugares del mundo, que afectan el desempeño académico de los estudiantes. Este documento se organiza en dos sesiones, determinantes a nivel mundial y determinantes a nivel colombiano; cada sesión clasifica las investigaciones de acuerdo a los factores del estudiante, de la escuela y las características organizacionales y políticas. A pesar de existir muchas investigaciones sobre el tema, aún no se ha llegado a un consenso general sobre cómo determinar los factores del rendimiento académico, pues en la realidad son muchas las características del individuo, la escuela o el sistema que se relacionan entre sí de diferente manera y pueden afectar el logro estudiantil.
\end{abstract}

Palabras clave: educación, factor colegio, factor estudiante, pruebas estandarizadas, rendimiento académico. 


\title{
STATE OF THE ART OF ACADEMIC ACHIEVEMENT IN SECONDARY EDUCATION
}

\begin{abstract}
This document shows the state of the art of the determinants of academic achievement in secondary education, taking into account the main research conducted, both nationally and internationally, about the factors that influence school achievement of students. With this paper, we seek to establish the main variables which affect the academic achievement of students in Colombia as well as in some parts of the world.

This document is organized in two sessions: world and Colombian determinants; each session classifies research according to the factors of the student, the school and organizational and political characteristics. Although there is much research on the topic so far it has not been possible to reach a consensus on how to determine the factors of academic achievement, because in reality many characteristics of the individual, of the school or of the system relate to each other differently and can affect student achievement.
\end{abstract}

Key Words: Education, school factor, student factor, standardized tests, academic achievement.

\section{ESTADO DA ARTE DO DESEMPENHO ACADÊMICO NO ENSINO MEDIO}

\begin{abstract}
RESUMO
Este documento mostra o Estado da arte dos determinantes do rendimento escolar no ensino medio, tendo em conta às principais pesquisas realizadas, tanto a nível nacional como internacionalmente, sobres os fatores que influenciam o desempenho escolar dos estudantes. Com este trabalho, se procura estabelecer as principais variáveis, tanto na Colômbia e em alguns lugares do mundo, afetand o o desempenho acadêmico dos estudantes. Este documento está organizado em duas sessões, determinantes a nível mundial e determinantes a nível colombiano; cada sessão clasifica as pesquisas de acordó a os fatores do estudante, da escola e das características organizacionais e políticas. Embora haja muitas pesquisas sobre o tema, ainda não se chegou a um consenso geral sobre os fatores determinantes no desempenho acadêmico, porque na realidade são muitas as características do indivíduo, a escola ou o sistema que se relacionam uns com os outros de forma diferente e podem afetar o desempenho acadêmico.
\end{abstract}

Palavras Chave: educação, fator colégio, fator estudante, testes padronizados desempenho acadêmico. 


\section{INTRODUCCIÓN}

El objetivo de cualquier sociedad del mundo es buscar un crecimiento económico sostenible en el largo plazo, interesada siempre por el mejoramiento del bienestar de toda su población. Algunos autores enfatizan que el crecimiento se genera en el seno de la economía bien por el capital humano (Lucas, 1998), por la existencia de rendimientos constantes a escala en la función de producción (Rebelo, 1991), por las externalidades tecnológicas (Romer, 1986), o por la ausencia de distorsiones en el mercado (Easterly, 1993). Lucas propone que el factor fundamental para lograr un aumento continuo del producto, en el largo plazo, es la cualificación del capital humano: a mayores años de estudio, mayor será la producción per cápita (productividad).

La educación, como proceso cultural, es una manifestación y/o herramienta fundamental de la cultura y del proceso de desarrollo de las instituciones en las distintas comunidades, etnias y grandes poblaciones organizadas. La educación no sólo es crucial en el proceso biológico de la hominización, sino que es la más poderosa herramienta social para la humanización; por esta razón, algunos pueblos han prevalecido sobre otros a lo largo de la historia de la humanidad, porque presentan mayores y efectivos accesos a la educación (Diamond, 1997).

En general, la educación se analiza desde tres perspectivas: 1) cobertura, 2) eficiencia y 3) calidad. Este trabajo se ubica en el último punto, pero no abarca su concepto en general, sino se limita al tema del rendimiento académico. Por esto, en este documento se pretende realizar una revisión literaria acerca de los determinantes de los factores que influyen en el rendimiento académico en la educación media, primaria y secundaria, tanto a nivel mundial como a nivel nacional. El análisis se realizó teniendo en cuenta investigaciones internacionales y nacionales sobre el tema, donde los resultados se clasificaron en dos grupos: por un lado los resultados de las investigaciones internacionales y, por el otro, los resultados de las investigaciones nacionales. Al mismo tiempo, cada grupo se subdividió en tres categorías más: 1) las características del estudiante, 2) las características de la escuela y 3 ) las características organizacionales y políticas. Es importante resaltar que este es un artículo de revisión, pues pretende exponer, desde diferentes perspectivas, los factores que determinan el rendimiento académico, mas no tomar postura alguna sobre los diferentes enfoques aquí expuestos. 


\section{EVIDENCIA INTERNACIONAL DE LOS FACTORES DETERMINANTES DE LOS RESULTADOS DEL APRENDIZAJE}

El aprendizaje estudiantil depende de muchos factores que, en algunos casos, no presentan relación, pero que, en la práctica, pueden influir; estos elementos van desde la educación del padre y los valores de la sociedad hasta la infraestructura escolar y el calendario agrícola. El último elemento parecería curioso, pero, si se piensa en una región rural, que económicamente depende de la agricultura, en épocas de cosecha toda la población, además de niños y docentes, se dedican a la recolección de los productos, bien sea por escasez de mano de obra, por necesidades económicas o porque el costo de oportunidad de hacer cualquier otra actividad es mayor (altas remuneraciones).

En este sentido, las políticas educativas dirigidas a mejorar los resultados escolares sólo pueden atender unos pocos de estos factores. Históricamente, las políticas educativas se dirigen a ofrecer insumos fácilmente cuantificables a los colegios y los sistemas escolares, tales como dinero, infraestructura, libros de texto, etc., pero dotar al sistema educativo de esta clase de insumos no garantiza necesariamente un mayor aprendizaje, ya que estos recursos controlables, de fácil rastreo y medición, visibles y viables políticamente, pueden tener efectos muy pequeños sobre el logro estudiantil (Vegas y Petrow, 2008).

Al estudiar la bibliografía de la investigación sobre los factores que determinan el rendimiento académico, Vegas y Petrow (2008) identifican una serie de variables asociadas a los estudiantes, las escuelas y las instituciones, que interactúan entre sí en un contexto económico, político y social que afecta el aprendizaje escolar (ver Gráfica 1).

A continuación se muestra una revisión bibliográfica de la evidencia internacional acerca de los factores determinantes de la calidad de la educación, con énfasis en los estudios que utilizan datos internacionales de evaluación, seguida de una descripción de la base de investigación colombiana.

\subsection{Características del estudiante}

Desde la publicación del Informe Coleman, en 1966, en los estudios sobre calidad educativa se encontró que las condiciones socioeconómicas de los estudiantes y sus antecedentes familiares causan los mayores efectos sobre el rendimiento académico; Coleman, un famoso sociólogo norteamericano, es el inspirador de esta nueva oleada de investigaciones que dan mayor importancia a las características individuales del estudiante y su familia. Estudios posteriores a Coleman (en Inglaterra, el informe Plowden y, en Francia, el estudio longitudinal 1962-1972, que condujo el Institut National d'Études Démographiques (NED)) mostraron de forma clara el peso del origen social sobre los destinos escolares; 
así, se llegó a la misma conclusión de Coleman. Estos resultados motivaron a muchos a decir que "la escuela no importa" (Muñoz y Murillo, 2001).

Gráfica 1. Elementos que Intervienen en el Aprendizaje Estudiantil.

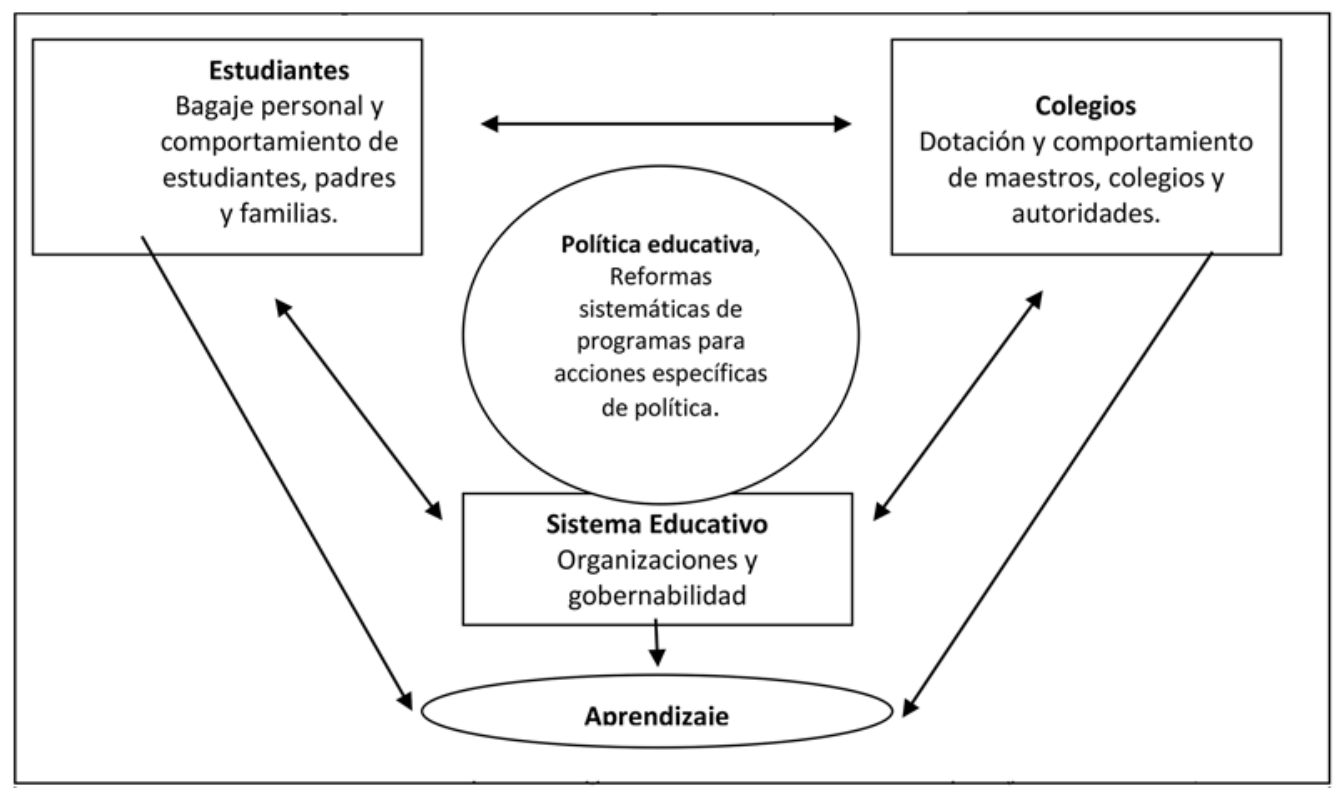

Fuente: Emiliana Vegas y Jenny Petrow (2008). Incrementar el Aprendizaje Estudiantil en América Latina (Washington D.C.: Banco Mundial/Mayol Ediciones, 2008), 69.

Las condiciones socioeconómicas, el ingreso, los años de escolaridad de los padres, la ocupación del padre, los recursos educativos del hogar, entre otros, son las variables que tradicionalmente se han utilizado para expresar las condiciones socioeconómicas y antecedentes familiares de los estudiantes, pero es claro que estas variables por sí solas no pueden explicar la dinámica no observable de los hogares, pues, por lo general, ocultan información de lo que realmente pasa en el hogar, así como la participación y motivación de los padres en el apoyo de la educación de sus hijos. Otro grupo de variables, como la edad, el sexo, la salud, la capacidad innata, la alfabetización temprana, las primeras experiencias escolares, entre otros, que por general las políticas públicas pueden influir, han mostrado tener algún nivel de asociación con el logro escolar y un impacto especialmente de largo plazo.

Según Vegas y Petrow (2008), la edad de entrada a la educación primaria puede afectar la trayectoria de un niño en el sistema educativo y sus logros en él; pero esta correlación tiene diferentes implicaciones en los países desarrolla- 
dos y en desarrollo. En Estados Unidos, los padres tienden a retardar la entrada de sus hijos a la escuela primaria con el fin de aumentar sus oportunidades de éxito en el colegio; en países en desarrollo, por su parte, la entrada retardada al colegio se debe a factores económicos y sociales. En América Latina, los menores ingresos de los padres se asocian con la inscripción escolar tardía (McEwan, 2006); por ejemplo, en Guatemala, el $20 \%$ de los niños inscritos en primaria lo hacen después de la edad oficial de siete años.

Angrist y Krueger (1991) sostienen que los costos de la entrada retardada a la educación pueden, en general, incluir un menor logro educativo para los estudiantes que abandonan más pronto, pues llegados a la edad promedio de terminación de estudios desertan sin haberlos culminado.

Otro grupo de investigaciones sostienen que el acceso a la escolaridad antes de la primaria mejora los resultados del aprendizaje estudiantil y reduce las desigualdades en la educación primaria y secundaria; Carneiro y Heckman (2003); Cunha, Heckman, Lochner y Masterov (2005); Berlinski, Galiani y Gertler (2006), estudiaron los efectos que tiene la educación preescolar (o educación de la primera infancia) en el rendimiento académico de los estudiantes de primaria en la Argentina, al aprovechar el Programa masivo de construcción preescolar, que es relativamente alto para el país, pero que carece de calidad. Al utilizar datos de la evaluación nacional y del Programa de construcción escolar de educación, los autores encontraron que "la asistencia preescolar a las edades de entre tres y cinco años aumenta el desempeño en lenguaje y matemáticas en 4,5 a 6,0 puntos $(0,23$ a 0,33 desviaciones estándar) sin muchas diferencias entre géneros; además, el efecto de los puntajes en pruebas en tercer grado de haber asistido a preescolar es el doble para estudiantes de antecedentes pobres con respecto a los de antecedentes no pobres".

Por otro lado, la condición socioeconómica es un factor importante para los resultados de aprendizaje o rendimiento escolar; en la gran mayoría de los estudios que consideran las características individuales, se encontró que la condición socioeconómica del padre, o más concretamente del hogar al que pertenece el individuo, resultó ser significativa. Hanushek y Luque (2003) utilizan los datos de la TIMSS para comparar el rendimiento de los sistemas de educación para países de diferentes niveles de desarrollo económico, al recurrir a funciones de producción educativas; los resultados muestran que los países de alto y bajo nivel de desarrollo presentan problemas generales en la utilización eficiente de los recursos; de manera específica, concluyen que los antecedentes familiares ejercen una fuerte influencia en el resultado escolar; en consecuencia, los estudiantes de familias desfavorecidas, en términos económicos, presentan desempeños inferiores a estudiantes de familias con ingresos medios y altos. 
Woessmann (2005) valida estos resultados para Argentina y Colombia, en un estudio que se realizó y al utilizar la Base de datos del estudio internacional de progreso en comprensión lectora de la IEA (PIRLS), que puso a prueba el rendimiento de lectura de estudiantes de cuarto grado en el año 2001. Las muestras representativas a nivel nacional tienen 3300 estudiantes en la Argentina y 5131 estudiantes en Colombia; en este trabajo, se estimó la relación entre los antecedentes familiares, características de la escuela y el logro del estudiante en la escuela primaria para Argentina y Colombia, en el que se encontró una fuerte relación entre los resultados académicos y los antecedentes familiares del individuo (mucho más fuerte en Argentina que en Colombia), y una débil relación entre las características institucionales de la escuela y el rendimiento académico.

La ocupación y la educación de los padres también son variables importantes en la determinación de un mayor resultado escolar; de hecho, dichas variables se utilizan a menudo como variables de remplazo para las condiciones socioeconómicas ${ }^{1}$. Fuchs y Woessmann (2005) encontraron fuertes correlaciones entre la ocupación y la educación de los padres y los puntajes en las pruebas de lectura en mayor proporción y, luego, en matemáticas, pero, según los autores, estos resultados pueden variar, al depender del contexto. Por otro lado, Fertig y Schmidt (2002) utilizaron datos individuales de PISA 2000; analizaron cómo se relacionan los resultados en la prueba de lectura con la información individual y los antecedentes familiares y con las características de la escuela; encontraron que la educación materna tiene un mayor efecto en el aprendizaje general del niño, pero que la educación paterna es más importante cuando se alcanza el nivel de educación terciaria.

\subsection{Características de la escuela}

La escuela, como uno de los elementos importantes en el proceso de aprendizaje de los individuos, afecta, sin lugar a dudas, el resultado escolar de los estudiantes; específicamente, factores como su dotación y comportamiento, en especial el comportamiento de los docentes afecta considerablemente la manera como aprenden los estudiantes. Las investigaciones, en este sentido, se interesan por comprender cómo afectan exactamente los factores referentes al colegio el resultado escolar mediante el examen de las diferencias en salones de clase, colegios, municipios y países; se realizaron estudios de distintas variables, como el tamaño del grupo, los materiales, el tiempo empleado en el colegio, la calidad de

1. En este sentido son varias las investigaciones que encuentran una correlación positiva entre el resultado académico y el nivel educativo o la ocupación de los padres (Casassus y otros, 2000; OCDE, 2001a; Willms y Somers, 2001; Fertig y Schmidt, 2002; Hanushek y Luque, 2003; Woessmann, 2003, 2004, 2005; Fuchs y Woessmann, 2004). 
los docentes, el comportamiento, su conocimiento y metodología de enseñanza, las características personales, como nivel de educación, experiencia laboral, etc.

Según Sanders y Rivers (1996), las características y el comportamiento del personal, en especial de los docentes, influyen de manera considerable en el aprendizaje estudiantil; el impacto de un docente en el resultado escolar del estudiante es acumulativo y de larga duración. Un docente ineficaz reduce potencialmente el desempeño de un estudiante durante años, pero varios docentes con las mismas características multiplican el efecto. Algo claro es que determinar las características y comportamientos de los docentes eficaces o ineficaces es un tema complejo, en especial debido a la escasez de información, en particular en los países en desarrollo, por tal razón son pocas las experiencias que avalan el impacto de variables específicas del docente sobre el resultado escolar.

Darling (2000), al utilizar datos de una encuesta de política para todos los Estados Unidos, encuestas de personal docente, y la Evaluación nacional de progreso educativo para los años 1993-1994, examina la relación entre las características del docente y otros insumos de la escuela, y el rendimiento académico de los estudiantes; los resultados sugieren que las políticas de inversión dirigidas a aumentar la calidad de los docentes pueden asociarse con mejoras en el rendimiento estudiantil. El nivel de formación de los docentes y la experiencia son las variables que presentan las correlaciones más altas con el logro estudiantil, especialmente en lectura y matemáticas, tanto antes como después de controlar el estado de pobreza y lenguaje del individuo. Los docentes con menor experiencia son los menos efectivos, en especial durante los primeros tres años de su carrera. Estos resultados los apoyan Clotfelter, Ladd y Vigdor (2007) en Carolina del Norte, quienes, al utilizar una Base de datos de todos los docentes y estudiantes del Estado durante un periodo de diez años, concluyeron que la experiencia del docente, sus puntuaciones en las pruebas y su licenciamiento regular aumenta el logro de los estudiantes en mayor medida en matemáticas y un poco menos en lectura.

Hanushek y Woessmann (2007), en su libro Education quality and economic growth, concluyen que efectivamente la calidad educativa, y no sólo el acceso a la educación, afecta el crecimiento económico; por lo tanto, se deben incentivar acciones que propendan por una mayor calidad educativa; los autores identifican que la calidad de los profesores es un ingrediente fundamental para el rendimiento académico; sin embargo, se resalta que las diferencias presentadas entre los profesores, que favorecen un mayor resultado académico, no se deben a las características individuales de cada profesor, tales como formación docente. Estos autores también observan que algunas medidas, como disminuir el número de estudiantes por clase, incrementar los sueldos de los profesores, aumentar el gasto de las escuelas, entre otras, impactan poco sobre el rendimiento de los es- 
tudiantes cuando la estructura general institucional permanece inalterada, pero esto no quiere decir que los recursos nunca tienen un impacto sobre el resultado académico, pues todo depende del contexto; por ejemplo, materiales como los libros tienen un impacto confiable sobre las escuelas menos desarrolladas ${ }^{2}$.

Las tecnologías de información y comunicación (TIC) tienen gran potencial para mejorar los procesos de aprendizaje. Estos resultados se han probado tanto en países desarrollados como en desarrollo; su efecto depende de cómo se utilicen y los tipo de actividades que pueden reemplazar. Asociado a las TIC viene el alfabetismo en computación que, por lo general, es un requisito básico de muchos trabajos, y hace que fuese importante la capacitación en destrezas en computación. Según Hepp y otros (2004), la inversión en TIC en la educación se considera como una forma primaria de preparar a las personas para las nuevas necesidades del mercado laboral y permitir que regiones rezagadas desarrollen las competencias necesarias para igualar las oportunidades de acceso al empleo.

La OCDE, en el 2006, realizó un estudio, con utilización de los datos del PISA 2003, con el objetivo de determinar si los estudiantes están preparados para un mundo tecnológico; se encontró que los estudiantes de 15 años de edad que tuvieron acceso a computadores en un periodo de tiempo (más de 5 años) obtuvieron promedios por encima de la media de la OCDE en matemáticas, mientras que los estudiantes con menor experiencia en computadores tuvieron promedio por debajo de la media de la OCDE, lo que sugiere que la exposición constante a computadores en un periodo largo de tiempo es un factor importante en el aprendizaje. Después de controlar las condiciones socioeconómicas, el estudio concluye que los computadores en el hogar generalmente tienen un mayor efecto positivo que los computadores en el colegio. Sorprende un poco que los estudiantes que usaron el computador por una cantidad de tiempo moderado (una vez por semana a una vez por mes) obtuvieran mejores resultados que estudiantes que lo utilizaron frecuentemente.

En el caso de Corea, un punto importante de la política educativa ha sido la apuesta por la introducción de las TIC en la educación, por lo que el e-learning (aprendizaje electrónico) se está impulsando en todos los niveles educativos como estrategia nacional para integrar a la población en la sociedad del conocimiento y construir una comunidad educativa de aprendizaje on-line, a lo que se denominó el séptimo currículo nacional, aprobado en 1997. Según González (2011), desde entonces las TIC comenzaron a ganar importancia en los Planes de estudio y en la Integración curricular, lo que provocó cambios en la organización

2. Los textos constituyen uno de los pocos insumos que, se ha demostrado consistentemente, causan un impacto fuerte en el aprendizaje estudiantil (Jamison y otros, 1981; Hayneman, Jamison y Montenegro, 1984; Lockheed y Verspoor, 1991; Harbison y Hanushek, 1992). 
y la planificación de los procesos de enseñanza/aprendizaje. Algunas de las innovaciones en TIC para la educación, desarrolladas por el gobierno coreano, son:

- El EBS: que consiste en una infraestructura de retransmisión pública de los servicios de televisión, radio e Internet vía satélite, que proporciona no sólo contenidos educativos sino también cursos de preparación del examen de acceso a la universidad.

- EDUNET: es un servicio de información educativa que le permite a los ciudadanos, acceder a diferentes fuentes de información sobre temas educativos, además de establecer una comunidad educativa on-line. También favorece la creación de comunidades de aprendizaje e intereses generales, lo cual facilita el intercambio de experiencias educativas y el intercambio de información (González, 2011).

- CHLS: es un tipo de educación voluntaria a través de una plataforma de aprendizaje on-line, donde los estudiantes de primaria y secundaria deciden participar. Según González (2011), el objetivo principal ha sido tratar de mejorar la calidad de la educación pública y reducir el gasto de las tutorías privadas a través de metodologías de trabajo más autónomas.

La política de TIC, en el Sistema educativo de Uruguay, tuvo un importante avance en el 2007, cuando el Gobierno implementó el Plan Ceibal, que otorga a cada niño y docente una computadora portátil y acceso a Internet de manera gratuita (modelo 1 a 1). Según Vaillant (2013), el Plan Ceibal fue, desde sus orígenes, un proyecto de carácter socioeducativo impulsado por el gobierno como parte de una iniciativa nacional global orientada a eliminar la brecha digital, para favorecer la inclusión social, cuya finalidad primera fue la de universalizar el acceso a computadoras y a Internet desde el primer nivel de escolarización básica. Este programa permite mejorar la calidad educativa a través de la integración tecnológica en el aula y la familia, mediante cuatro líneas: niño-niño; niño-maestro; maestro-maestro y niño-familia-escuela. Hoy en día, el programa alcanzó la universalización y cobertura completa.

Al igual que en Uruguay, en Colombia existe el programa Computadores para Educar; aunque sin la universalidad y cobertura que el Plan Ceibal, es un proyecto que genera equidad a través de las tecnologías de la Información y las comunicaciones, al fomentar la calidad de la educación con un modelo sostenible. El programa incluye la entrega de equipos de cómputo y la formación a los docentes para su máximo aprovechamiento, con el propósito de poner a las TIC al alcance de las comunidades educativas, especialmente en las sedes educativas públicas del país. En los últimos años, más de 7 millones de niños y jóvenes, de 
más de 35 mil sedes educativas, las más apartadas y rurales del país, experimentan una "Vida Digital" gracias a las posibilidades que les brinda la tecnología ${ }^{3}$.

Finalmente, otras de las variables relativas al colegio, que se estudian con mayor frecuencia en las funciones de producción de educación, es el tamaño del grupo y las razones estudiantes-docente ${ }^{4}$; aunque la evidencia sobre los efectos del tamaño del grupo y la relación estudiante-docente en el resultado escolar no es concluyente, se registran aportes como los de Willms y Somers (2001), quienes emplearon un modelo de regresión jerárquica para examinar las relaciones entre los logros en lenguaje y matemáticas, los antecedentes familiares y las diversas prácticas y políticas escolares; los análisis incluyen datos para 13 países de América Latina, con muestras de aproximadamente 100 escuelas en cada país. Los autores encontraron que la relación entre los resultados escolares y los antecedentes familiares varía entre países; concluyen que la relación resultado escolar $v s$. la razón estudiantes-docente es estadísticamente significativa en casi todos los países, pero empíricamente carece de importancia; por lo tanto, esta correlación se muestra negativa, pero el efecto es aún muy pequeño. La OCDE (2001), en los resultados de la aplicación de la prueba PISA 2000, encontró que cuando la razón estudiantes-docente aumenta por encima de 25 estudiantes, el puntaje promedio en todas las áreas evaluadas por el PISA presenta un menor desempeño, es decir, que los salones de clases con más de 25 estudiantes presentan menores rendimientos que salones con menos estudiantes.

\subsection{Características organizacionales y políticas}

Algunas investigaciones recientes consideran a la estructura organizativa de los países y las instituciones como factores determinantes del resultado escolar. La autonomía en la toma de decisiones sobre el financiamiento, los gastos, la contratación y el despido de docentes, las decisiones pedagógicas y los Planes de estudios, son algunos de los factores que determinan el desempeño académico.

Fuchs y Woessmann (2005) sugieren que los factores organizativos pueden explicar hasta un $25 \%$ de las variaciones en los puntajes entre los países; la mayor autonomía escolar en la administración del personal y las decisiones sobre procesos (contratación de docentes, selección de textos, asignaciones presupuestales dentro de los colegios) se correlacionan fuertemente con un mejor desempeño estudiantil.

3. Cifras tomadas de: ¿Qué es computadores para educar?, http://www.computadoresparaeducar.gov.co/ inicio/ ?q=node/27

4. Los siguientes autores, y otros, han considerado la variable tamaño del grupo en sus análisis: Hanushek, 1995, 2003; Pritchett y Filmer, 1997; Hanushek y Kimko, 2000; Woessmann, 2001 y Pritchett, 2004; Fertig y Schmidt, 2002; Woessmann y West, 2002, 2006; Hanushek y Luque, 2003, y Woessmann, 2003, 2005 . 
Vegas y Petrow resumen las variables organizativas y políticas que se relacionan positivamente con el logro de los estudiantes, que permiten establecer las diferencias entre países, variables que son:

- Control centralizado de asuntos curriculares y presupuestales.

- Administración de colegios a nivel intermedio (y no a nivel central).

- Autonomía escolar en cuanto a decisiones sobre procesos y de personal.

- Incentivos para los docentes individualmente.

- Métodos de enseñanza seleccionados por los docentes.

- Evaluaciones nacionales.

- Participación de los padres de familia.

Otras investigaciones encuentran que las diferencias existentes en las instituciones de enseñanza explican, a su vez, las grandes diferencias internacionales en el rendimiento de los estudiantes en las pruebas de logro cognoscitivo; es decir, estas diferencias internacionales no las causan las diferencias de los recursos de escolaridad en sí, sino se deben principalmente a las diferencias en las instituciones de enseñanza. Otro aspecto importante es el nivel de autonomía curricular, que puede afectar el aprendizaje de los estudiantes: "el análisis de las evaluaciones internacionales indica que los estándares centralizados de planes de estudio emparejados con la flexibilidad local en la implementación curricular se asocia con mejores puntajes en las pruebas" (Woessmann, 2003; Hanushek y Woessmann, 2007).

King y Ozler (2000) examinan el impacto de la Reforma de la autonomía escolar en Nicaragua sobre el aprendizaje estudiantil, al utilizar una función de producción educativa; la autonomía en la toma de decisiones se relaciona positivamente con la puntuación de los estudiantes de las escuelas primarias. En efecto, la autonomía en la selección de docentes, los sueldos, los incentivos, su control y evaluación, pueden elevar el desempeño académico de los estudiantes.

Filmer y Eskeland (2002) encuentran los mismos resultados para Argentina: "la autonomía de los colegios de primaria se asocia con mejor desempeño estudiantil"; esto se realizó mediante la aplicación de un cross-section para los grados $6^{\circ}$ y $7^{\circ}$. Otro de los hallazgos radica en la participación de los padres de familia en la administración del colegio (juntas directivas, consejo de padres de familia, etc.), que afecta el resultado escolar, pero sólo a través de la mediación del efecto de la autonomía escolar, pues en estas circunstancias los padres pueden llegar a tener cierto grado de decisión en el colegio. Estos resultados son comunes en todas las sub-muestras que los autores realizaron: niños de hogares de muy escasos recursos, hijos de madres sin formación en la escuela, escuelas públicas, escuelas privadas, familias con riqueza media, etc. 
La descentralización también puede aumentar las desigualdades dentro de los resultados académicos de los países; en estudios realizados en Brasil, Honduras, Nicaragua, El Salvador y Argentina, se encontró que mientras la descentralización causó, en promedio, un impacto positivo sobre el desempeño académico de los estudiantes a nivel nacional, a nivel más desagregado (municipio, región, provincia) los resultados mostraron una mayor desigualdad; por ejemplo, en municipios de bajos recursos de Argentina, que tenían una débil capacidad institucional antes de la Reforma de descentralización, los puntajes de los estudiantes en las pruebas cayeron hasta un 15\%, después de la Reforma (Galiani, Gertler, y Schargrodsky, 2005).

El sector privado juega un papel importante en la educación de los latinoamericanos; según Wolff, Navarro y González (2005), América Latina tiene grandes antecedentes de provisión privada de escolaridad, de manera más consistente en los niveles de educación preescolar, secundaria y superior. La efectividad de las instituciones públicas versus privadas, la elección de colegio, el sistema de cupones y demás, y su relación con los resultados escolares, se incluyen en un debate actual y cadente entre los economistas de la educación de todo el mundo. Algunos investigadores sostienen que permitir a grupos no gubernamentales la provisión de los colegios, pero con financiación parcial o total del gobierno ${ }^{5}$, produce mayores resultados, pues los colegios privados son más eficientes y efectivos que los colegios públicos. Según ellos, "abrir todos los colegios a la competencia proporcionando cupones y aumentando la elección paterna mejora el aprendizaje tanto en los colegios públicos como en los privados". Por otra parte, también se argumenta que los colegios privados tienen mayor éxito porque retienen a los mejores docentes de acuerdo al desarrollo de las destrezas de los existentes; de esta forma, generan mayores resultados en los estudiantes, pero este mayor éxito se debe, entre otros aspectos, a la mayor supervisión en los colegios privados y el sistema de mentores de docentes nuevos, su capacidad de exigir que los docentes tengan educación de mejor calidad, su tendencia a atraer a docentes que realizan mayor esfuerzo y son independientes y su libertad de despedir a los docentes por desempeño deficiente (Ballou y Podgursky, 1998; Hoxby, 2003).

Otro grupo de investigadores, opositores a los anteriores, argumentan que efectivamente la elección del colegio es un método efectivo para mejorar el rendimiento académico, pero sólo en los grupos de estudiantes con altos niveles socioeconómicos, pues es evidente que el sistema de cupones conduce a una mayor categorización, donde los estudiantes "ricos" pueden elegir los mejores

5. Al estilo chileno, donde el Sistema educativo financia a la demanda, con asignación de boucher o cupones a los estudiantes, para que puedan escoger el colegio que quieran; si deciden entrar a un colegio privado, el cupón cubre parte de los costos laborales y el estudiante debe asumir el costo faltante. 
colegios, que, por lo general, son los privados, lo que deja a los estudiantes de la parte baja de la distribución del ingreso los demás colegios, por lo general públicos, de bajo rendimiento, pero esto se complementa con el poder de elección que tienen los colegios privados frente a los colegios públicos; los privados pueden seleccionar a sus estudiantes, lo que causa una mayor categorización por antecedentes de raza o grupos étnicos, socioeconómicos y culturales; por su parte, a los colegios públicos, al no poder elegir sus estudiantes, les corresponde la difícil tarea de atender a los niños más desfavorecidos, con menores recursos de los que se tendrían disponibles en un sistema completamente público (González, Mizala y Romaguera, 2004; Hsieh y Urquiola, 2003).

\section{EVIDENCIA COLOMBIANA DE LOS FACTORES DETERMINANTES DE LOS RESULTADOS DEL APRENDIZAJE}

La evidencia empírica disponible sobre los factores determinantes del aprendizaje en Colombia refuerza gran parte de los hallazgos de investigaciones aplicadas en distintas partes del mundo. En los estudios colombianos, prima el uso de los datos de las evaluaciones nacionales aplicadas fundamentalmente por el ICFES para todos los niveles de educación, que buscan, por un lado, esclarecer los factores determinantes del aprendizaje, y, por el otro, evaluar ciertos programas y políticas. Para tratar de seguir con la misma estructura de la evidencia internacional, las investigaciones a nivel nacional se agruparon en las mismas tres categorías.

\subsection{Características del estudiante}

Las investigaciones sobre los factores determinantes del aprendizaje, en Colombia, por lo general consultan no solamente las variables que tienen que ver con las características del estudiante, sino también, y de paso, las características de la escuela y las organizacionales y políticas.

Dentro de las variables de los estudiantes, que presentan mayor relación con el resultado escolar, están los antecedentes socioeconómicos; Fuchs y Woessmann (2005) encuentran una fuerte y significativa correlación entre la situación socioeconómica y el rendimiento educativo de los estudiantes de primaria en Colombia; después de un control por los ingresos, tener libros en el hogar genera un impacto positivo, pero pequeño, sobre las calificaciones de lectura de los alumnos de grado $4^{\circ}$. Del mismo modo, encuentran que los antecedentes familiares influyen considerablemente en aspectos específicos como el desarrollo del alfabetismo; otra de las conclusiones de la misma investigación es que el ingreso económico familiar se relaciona significativamente con el rendimiento académico. 
Caro (2000), en un estudio que realizó para la ciudad de Bogotá, determinó los factores asociados al logro académico de los estudiantes de $3^{\circ}$ y $5^{\circ}$ de primaria. Al igual que Fuchs y Woessmann, encuentra una fuerte correlación entre los antecedentes socioeconómicos y el resultado escolar; además, encuentra que el ausentismo y el trabajo infantil tienen grandes efectos negativos en el desempeño académico de los estudiantes. Piñeros y Rodríguez llegaron a resultados similares en un estudio publicado en 1999, que trabajó con todos los bachilleres de Colombia que presentaron las pruebas Saber 11; los autores sostienen que la distancia del viaje entre la escuela y el lugar de residencia tiene también efectos negativos en el desempeño en el Examen de Estado Nacional; dicen, además, que el nivel socioeconómico del estudiante repercute positivamente en el puntaje de las pruebas, pero su impacto se hace particularmente mayor en idiomas que en matemáticas o ciencias. Otro aspecto que afecta el desempeño académico, según los autores, es la continuidad en la escuela; mantener a los estudiantes en la misma escuela durante sus estudios secundarios afecta positivamente el resultado escolar, debido a que esta continuidad actúa para reducir al mínimo los costos de transición, que pueden tener efectos negativos sobre el puntaje en las pruebas.

En Calidad de la educación y Rendimiento Académico en Bogotá, un estudio realizado por Gaviria y Barrientos en 2001, se sostiene que la calidad de la educación secundaria es un factor clave en el logro socioeconómico de las personas y, por lo tanto, un factor determinante en el acceso a las oportunidades y la movilidad social; estos autores estudiaron los efectos del entorno familiar y las características del plantel sobre el rendimiento escolar en Bogotá, con base en los resultados de las Pruebas Saber 11 para el año 1999. Tres conclusiones principales se desprenden del trabajo: 1) la educación de los padres tiene un efecto sustancial sobre el rendimiento académico, 2) el efecto de la educación de los padres se transmite principalmente a través de la calidad de los planteles educativos, y 3) la incidencia de los planteles sobre el rendimiento supera la incidencia conjunta de las características familiares y es mayor que la observada en otros países; según ellos, estas tres premisas sugieren que la existencia de restricciones en el acceso a la buena educación constituye un factor muy importante de inmovilidad social en Colombia.

Chica, Galvis, y Ramírez (2010), al utilizar los resultados en las áreas de lenguaje y matemáticas de las pruebas Saber 11, para el segundo semestre del año 2009, identificaron los determinantes del rendimiento académico en Colombia, donde se utilizó un modelo Logit Ordenado Generalizado; los autores comprobaron que las variables socioeconómicas juegan un papel primordial en la calidad de la educación; tener condiciones económicas favorables afecta en gran medida la probabilidad de obtener un puntaje medio y alto tanto en matemáticas como 
en lenguaje, lo que puede explicarse en razón a que un entorno socioeconómico favorable le permite al estudiante contar con las condiciones esenciales para dedicarse a sus estudios: una buena alimentación, transporte, infraestructura y herramientas informáticas hacen parte de estas condiciones.

En este sentido, variables, como tener computador en casa, el nivel de ingreso y el nivel de escolaridad de los progenitores, se relacionan positiva y significativamente con el logro escolar: padres con mayor nivel educativo pueden apoyar favorablemente el proceso de enseñanza y aprendizaje de sus hijos; este mayor nivel educativo, por lo general, se asocia con un mayor nivel de ingreso, necesario para adquirir la infraestructura suficiente, como computadores, libros, etc., que garantice un nivel alto de rendimiento académico. Los autores también concluyeron que, por lo general, los hombres se desempeñan mejor en el área de matemáticas, mientras que las mujeres lo hacen mejor en lenguaje.

\subsection{Características de la Escuela}

La escuela, espacio común sobre el que se aplica la mayoría de las políticas educativas, adquirió un creciente interés en las investigaciones de rendimiento académico y calidad educativa del país, con el propósito de determinar qué elementos se deben promover en las reformas políticas que buscan mejorar la calidad de la educación.

Son muchos los estudios que muestran que las escuelas o instituciones educativas tienen efectos sobre los resultados del aprendizaje escolar. Piñeros y Rodríguez (1999) encontraron que "las escuelas tienen un efecto pequeño, pero considerable, sobre el logro académico, teniendo entre $15 \%$ y $18 \%$ de la varianza entre estudiantes en escuelas privadas y $12 \%$ y $16 \%$ en escuelas públicas". Otro de los hallazgos del estudio se refiere a las grandes diferencias de desempeño en las pruebas que existe entre y dentro de las escuelas especialmente del sector privado, lo que puede deberse a las diferencias en dotación física de los establecimientos educativos. La infraestructura académica y los materiales didácticos muestran una correlación positiva con el logro escolar; por tanto, los laboratorios de ciencias, las bibliotecas, las instalaciones deportivas, tienen un efecto estadística y empíricamente significativo y positivo sobre el rendimiento académico de los estudiantes; es decir, un suministro adecuado de insumos repercute positivamente en el logro escolar. Los autores también sostienen que los estudiantes matriculados en escuelas de jornada completa muestran un rendimiento significativamente mayor que los estudiantes matriculados en escuelas con jornadas de medio día.

Las características de los docentes, en especial el nivel educativo y la experiencia, tienen impactos positivos sobre el rendimiento académico, o al menos 
así lo muestra la creciente evidencia sobre el tema, que, a su vez, recalca sobre las dificultades al describir las características de los buenos profesores; por esta razón, tanto los estudios nacionales como internacionales, se han centrado en las características cuantificables de los profesores, donde se encuentra, en algunos casos, una correlación positiva con el logro escolar y en otros no existe tal relación (Hanushek y Woessmann, 2007), pero este efecto es significativo en investigaciones que utilizan pruebas nacionales.

Caro (2000) encontró que, en los estudiantes de $3^{\circ}$ y $5^{\circ}$ de Bogotá, la escolaridad promedio de los profesores se relaciona con diferencias de rendimientos entre escuelas. Por su parte, en investigaciones donde se utilizaron datos de evaluaciones internacionales, la relación entre la educación y la experiencia de los docentes y el puntaje promedio de dichas evaluaciones no es significativa; Fuchs y Woessmann (2005), al utilizar el PIRLS del 2001, dicen que no existe ninguna relación estadísticamente significativa entre el desempeño académico de los estudiantes y el poseer un título universitario y/o certificación en pedagogía por parte del docente; con respecto a la experiencia laboral del docente, tampoco se presenta dicha relación. Hanushek y Luque (2003) llegaron a conclusiones similares con el uso de resultados de las pruebas TIMSS del año 1995; los autores muestran que, en más de 40 países, incluido Colombia, el porcentaje de profesores con título universitario y el porcentaje de aquellos que reciben capacitación docente especializada, no presenta significancia estadística con los resultados de dicha prueba; por lo tanto, los docentes no influyen mucho en el desempeño de los estudiantes.

Chica, Galvis, y Ramírez (2010) encontraron que los estudiantes de jornada completa obtuvieron puntajes más altos en comparación con los estudiantes que asistían a otras jornadas, como las nocturnas, sabatinas y dominicales; este resultado es importante en el momento de establecer políticas de cobertura fundamentadas en la partición de la jornada y no en la creación de nuevas instituciones. Otro aporte interesante que los autores realizan, de acuerdo al tipo de población que atiende el colegio, es el hecho de que estudiar en establecimientos con población exclusivamente femenina o masculina disminuye la probabilidad de pertenecer a un nivel bajo de promedio.

López (2010), a partir de las pruebas nacionales del ICFES Saber 11, desde 1980 a 2009, estimó el porcentaje de la variabilidad del logro académico atribuible al plantel educativo dentro de un análisis multinivel discriminado por sector educativo; el autor sugiere que el porcentaje de la variabilidad del logro en las pruebas nacionales se explica por las características propias y relativamente constantes del colegio; fue, en estas tres últimas décadas, siempre menor para los colegios públicos en comparación con los colegios privados y para el total de colegios del país. Otro punto importante es el cambio de forma y fondo que 
sufrieron las pruebas de Estado después de la última década; estos cambios de metodología incidieron en los resultados de las pruebas, pues los puntajes promedios bajaron drásticamente, en especial en el sector público, lo que ha producido que el coeficiente del factor colegio bajase considerablemente en ambos sectores.

\subsection{Características institucionales y organizativas}

Las investigaciones recientes, desarrolladas en Colombia, consideraron como características institucionales y organizativas, esencialmente, la prestación del servicio educativo por parte de sector público y privado. Fuchs y Woessmann (2005), que estudiaron el desempeño en el PIRLS de Colombia y otros países en 2001, encontraron que los estudiantes de instituciones educativas cuyo programa de estudios lo establece una autoridad central, a partir de un programa de estudios nacional o regional, no tuvieron un rendimiento significativamente mejor que aquellas instituciones cuyo Plan de estudio se estableció de forma independiente, de acuerdo a las necesidades del entorno, lo que indica el éxito de la autonomía y las adaptaciones locales.

Mucha de la evidencia que se referenció anteriormente, tanto a nivel nacional como internacional, respalda la idea de que las instituciones educativas privadas presenten un mejor desempeño que las instituciones públicas; por tanto, pertenecer a una escuela privada aumenta la probabilidad de éxito en las distintas pruebas. Sin embargo, este tipo de conclusiones debe analizarse con cuidado ya que prevalece un problema importante de selección. Cox y Jiménez (1991) examinaron la eficiencia relativa de las escuelas públicas y privadas de Colombia y Tanzania; es decir, estudiaron el rendimiento de ambos sectores, al comparar entre los dos países; encontraron que los estudiantes de escuelas privadas tienen calificaciones mayores que los estudiantes de escuelas públicas, en ambos países; sin embargo, sostienen que los colegios privados tienden a reservarse el derecho de admisión; por tanto, los autores afirman que el sesgo de selección sí afecta la medición de las diferencias de logro público/privado.

Piñeros y Rodríguez (1999) llegaron a conclusiones similares, una vez controlado el efecto del nivel socioeconómico sobre el desempeño académico; así, los estudiantes de las escuelas públicas rinden mejor que los estudiantes de las escuelas privadas. Como evidencia de los resultados de estos estudios, la situación socioeconómica de los estudiantes juega un papel preponderante en la determinación del desempeño de los estudiantes de las escuelas tanto privadas como públicas.

Como se mencionó anteriormente, Gaviria y Barrientos (2001) encontraron que, entre las escuelas privadas, el nivel de educación promedio del docente y 
el número de estudiantes por docente se relacionan fuertemente y de manera positiva con el rendimiento escolar. Por su parte, en el sector público, no hay ninguna relación entre estas dos variables y el rendimiento académico; según los autores, los efectos de las características de la escuela sobre el rendimiento los media la estructura de incentivos que rige las relaciones entre los estudiantes, los profesores, los personajes públicos y los padres.

Mina (2004), en su investigación Factores asociados al logro educativo a nivel municipal, trató de distinguir cuáles son las variables que inciden en la calidad de la educación en 897 municipios de Colombia durante los años 1966-1999, a través de la estimación de una función de producción en educación, con una estructura de panel de datos; el autor encontró que el gasto en educación por niño (positivo) y la pobreza y desigualdad (negativo) de cada municipio se correlacionan significativamente con el rendimiento académico. Mina determinó que las políticas que buscan aumentar la cobertura educativa de la población no afectaron el rendimiento académico de los municipios con mayores niveles de urbanidad; en las zonas rurales, por el contrario, el aumento de la cobertura incidió negativamente sobre el rendimiento educativo de dichos municipios.

\section{CONCLUSIONES}

Tanto la escuela como las características propias del individuo, así como las características institucionales y políticas determinan el rendimiento académico de los estudiantes. En este sentido, el rendimiento académico es un concepto multidimensional determinado por un conjunto amplio de variables que se deben tener en cuenta en el momento de formular políticas educativas efectivas; naturalmente, la política sólo puede atender unos pocos de estos factores.

Es difícil identificar empíricamente cómo y en qué grado contribuyen las distintas variables en el aprendizaje estudiantil. Según el Banco Mundial (2006), los factores que influyen en el aprendizaje pueden afectar a los estudiantes de manera diferente, de acuerdo a sus características físicas, al sector socioeconómico, al género, entre otros; además, estas características pueden interactuar entre sí y producir resultados inesperados: "El impacto de los recursos en el aprendizaje estudiantil puede ser limitado, pues los recursos no se asignan necesariamente con el propósito de mejorar el aprendizaje estudiantil. Los colegios y sistemas escolares son lugares politizados en gran parte, en los que las decisiones se toman por distintas razones, de las cuales el mejoramiento del aprendizaje estudiantil puede ser sólo una de tantas".

La mayoría de las investigaciones utilizan funciones de producción de educación con el objetivo de medir las relaciones entre individuo, familia, colegio 
y características institucionales, por una parte, y las dotaciones y resultados del aprendizaje, por la otra. En este marco analítico, los Sistemas educativos se consideran como sistemas productivos, donde los insumos escolares interactúan entre sí para llegar a un resultado, como el aprendizaje estudiantil; sin embargo, aún no se ha llegado a un consenso en cuanto a cómo crear modelos precisos que identifiquen los determinantes del rendimiento académico; actualmente, aún continúan los debates acerca de qué insumos deben incluirse, cómo deben medirse y qué forma debe tomar la función de producción (Hedges, Laine y Greenwald, 1994); por lo tanto, la literatura sobre los efectos de los determinantes en el logro estudiantil es extensa, mas no concluyente.

A manera de síntesis, se puede comentar que los factores que influyen en el aprendizaje de los estudiantes son complejos y difíciles de medir, pues cada niño es distinto, así como también lo es cada salón de clases, cada colegio, comunidad y nación; no obstante, se pueden establecer algunas enseñanzas comunes de la investigación referenciadas en este documento.

Una buena infraestructura escolar, una planta docente con experiencia, formación en pedagogía y conocimientos en su disciplina, así como profesores con altos niveles de formación académica, influyen notablemente sobre el rendimiento académico de los estudiantes que pertenecen a entornos socioeconómicos menos desarrollados. La jornada académica, el número de estudiantes por docente y el tipo de bachiller, también determinan notablemente el puntaje promedio en las distintas pruebas estandarizadas aplicadas en los distintos países.

El nivel académico de los padres influye en el rendimiento académico de los estudiantes de todos los estratos socioeconómicos, pero su efecto es mayor en regiones con mayor nivel de desarrollo económico.

El acceso a las tecnologías de la información y la comunicación se ha convertido en una herramienta importante en el proceso educativo, que se usa con más frecuencia en los últimos años. Las prácticas académicas en el aula de clase han logrado un alto grado de innovación, con la incorporación especialmente de sistemas de computación, tableros inteligentes y demás, que han favorecido el rendimiento académico de los estudiantes, lo que se evidencia, en mayor medida, en colegios de carácter privado, quienes ofrecen una mayor cantidad de insumos escolares que benefician el rendimiento académico. En cuanto a los insumos escolares en casa, los estudiantes con computador en el hogar obtienen un rendimiento mayor que quienes no tienen computador; del mismo modo, el acceso a Internet favorece el desempeño académico de los estudiantes. 
Los hombres se desempeñan mejor en el área de matemáticas, mientras que las mujeres se desarrollan mejor en áreas como literatura, idiomas y ciencias. Del mismo modo, en algunos casos, los estudiantes con menor edad obtienen mayores rendimientos que estudiantes con edades muy por encima del promedio; esta diferencia se hace más notable al comparar los extremos de mayores y menores edades, lo que resulta evidente al suponer que los estudiantes de mayores edades y jornadas académicas especiales (como la nocturna o sabática), además de estudiar, realizan actividades laborales. Se debe aclarar que existe otro grupo de investigaciones que argumentan lo contrario con respecto a la edad, que citan parámetros como la motivación, la experiencia, el nivel socioeconómico y cultural.

La organización y administración de las instituciones educativas y los sistemas escolares influye en el aprendizaje de los estudiantes, concretamente en la igualdad de oportunidades, en especial en el acceso de los estudiantes. En algunos casos, la descentralización produjo resultados decepcionantes, al reducir el desempeño de los estudiantes en colegios o distritos de bajos recursos o mal administrados; no obstante, aspectos como la participación de los padres y la autonomía escolar son medidas efectivas para mejorar los resultados en las pruebas y alcanzar indicadores de calidad, tales como disminución en las tasas de repetición y deserción.

Si se quisiera comparar los determinantes del rendimiento académico en Colombia con los determinantes a nivel internacional, de acuerdo con los estudios analizados en este documento, se puede concluir que en el país predominan variables del factor colegio; al igual que en países con bajos niveles de ingreso (o menos nivel de desarrollo económico), infraestructura, docentes calificados y acceso a TIC, son algunas de las variables que predominan en países como Colombia. Por el contrario, en territorios con mayor grado de desarrollo económico, las variables de tipo familiar y personal presentan mayor impacto en el logro académico. 


\section{REFERENCIAS BIBLIOGRÁFICAS}

Angrist, Joshua D. y Alan B. Krueger. Does Compulsory Schooling Attendance Affect Schooling and Earnings? En: Quarterly Journal of Economics. Vol. 106, No. 4, (nov., 1991): 979-1014.

Ballou, Dalle y Michael Podgursky. Teacher Recruitment and Retention in Public and Private Schools. En: Journal of Policy Analysis and Management. Vol. 17, No. 3 (verano, 1998): 393-417.

Berlinski, Samuel; Sebastián Galiani y Paul Gertler. The Effect of Pre-primary Education on Primary-school Performance. Londres: Institute for Fiscal Studies, 2006 (Documentos de trabajo W06/04).

Caro, Blanca Lilia. Factores asociados al logro académico de los alumnos de $3^{\circ}$ y $5^{\circ}$ de primaria de Bogotá. Coyuntura Social. Fedesarrollo. No. 22, (may., 2000): 65-80.

Chica, Sandra Milena; Diana Maritza Galvis y Andrés Ramírez Hassan. Determinantes del rendimiento académico en Colombia. Pruebas ICFES - Saber 11ํ, 2009. En: Revista Universidad EAFIT. Vol. 46. No. 160 (2010): 48-72.

Clotfelter, Charles T.; Helen F. Ladd y Jacob L. Vigdor. How and Why do Teacher Credentials Matter for Student Achievement? Cambridge: National Bureau of Economic Research, 2010 (Documento de trabajo NBER 12828).

Coleman, James S. Equality of Educational Opportunity. Washington: US Government Printing Office, 1966.

Cox, Donald y Emmanuel Jiménez. The Relative Effectiveness of Private and Public Schools: Evidence from two Developing Countries. En: The Journal of Development Economics. Vol. 34, No. 1-2 (1991): 99-121.

Cunha, Flavio; James J. Heckman; Lance Lochner y Dimitry V. Masterov. Interpreting the Evidence on Life Cycle Skill Formation. Cambridge: National Bureau of Economic Research, 2005 (Documento de trabajo NBER 11331).

Darling-Hammond, Linda. Teacher Quality and Student Achievement: A Review of State Policy Evidence. En: Education Policy Analysis Archives. Vol. 8. No. 1, (en., 2000).

Diamond, Jared M. Guns, germs, and steel: the fates of human societies. Los Angeles: Debate Editorial, 1997.

Easterly, William. How much do distortions affect growth? En: Journal of Monetary Economics. Vol. 32 . No. 2, (1993): 187-212.

Fertig, Michael y Christoph M. Schmidt. The Role of Background Factors for Reading Literacy: Straight National Scores in the PISA 2000 Study. En: Institute for the Study of Labor (IZA) (ag., 2002) (Documento para discussion 545).

Filmer, Deon y Gunnar S. Eskeland. Autonomy, Participation, and Learning in Argentine Schools: Findings, and Their Implications for Decentralization. Washington, DC: Banco Mundial, 2002 (Documento de trabajo 2766).

Fuchs, Thomas y Ludger Wößmann. What Accounts for International Differences in Student Performance? A Re-examination using PISA Data, http://www.oecd.org/edu/school/programmeforinternationalstudentassess mentpisa/33680685.pdf.

Galiani, Sebastian; Paul J. Gertler y Ernesto Schargrodsky. School Decentralization: helping the Good Get Better, but Leaving the Poor Behind, file://D:/Documents\%20and\%20Settings/Administrador/Mis\%20 documentos/Downloads/SSRN-id2450613.pdf.

Gaviria, Alejandro y Jorge Hugo Barrientos. Determinantes de la calidad de la educación en Colombia. Bogotá: Departamento Nacional de Planeación/Dirección de Estudios Económicos, 2001 (Documento 159).

González Pérez, Alicia. Políticas educativas en Corea del Sur: buenas prácticas TIC en la sociedad del conocimiento". En: Análisis. Año 14, No. 40, (ab., 2011): 75-90.

González, Pablo; Alejandra Mizala y Pilar Romaguera. Vouchers, inequalities and the chilean experience, http:// www.ncspe.org/publications_files/Voucher_ChileOP94.pdf 
Hanushek, Eric A. The Economics of Schooling: Production and Efficiency in Public Schools. En: Journal of Economic Literature. Vol. 24, No. 3, (sept., 1986): 1141-1177.

Hanushek, Eric A. y Javier A. Luque. Efficiency and Equity in Schools around the World. En: Economics of Education Review, Elsevier. Vol. 22 (5), (oct., 2003): 481-502.

Hanushek, Eric A. y Wößmann, L. Education quality and economic growth. Washington D.C.: Banco Mundial, 2007.

Heckman, James J.; Alan B. Krueger y Pedro Carneiro. Inequality in America: What Role for Human Capital Policies? Cambridge: MIT Press, 2003.

Hedges, Larry V.; Richard D. Laine y Rob Greenwald. Does Money Matter? A Meta-analysis of Studies of the Effects of Differential School Inputs on Student Outcomes. En: Educational Researcher. Vol. 23. No. 3, (ab., 1994): 5-14.

Hepp K., Pedro; Enrique Hinostroza S.; Ernesto Laval M. y Lucio Rehbein. Technology in Schools: Education, ICT, and the Knowledge Society. Washington, DC: Human Development Network, Banco Mundial, 2004.

Hoxby, Caroline M. Does Competition among Public Schools Benefit Students and Taxpayers? En: The American Economic Review. Vol. 90, No. 5, (dic., 2000): 1209-1238.

Hsieh, Chang-Tai y Miguel Urquiola. When schools compete, how do they compete? An assessment of Chile's nationwide school voucher program (nov., 2004), http://www.columbia.edu/ msu2101/HsiehUrquiola\%28 2004\%29.pdf.

King, Elizabeth M. y Berk Özler. What's decentralization got to do with learning? Endogenous school quality and student performance in Nicaragua, http://citeseerx.ist.psu.edu/viewdoc/download?doi=10.1.1.17.6332\& rep=rep $1 \&$ type $=$ pdf.

López Mera, Silvio Fernando. El efecto colegio en Colombia: tres décadas de estudio. En: Revista Equidad y Desarrollo. No. 14, (2010): 85-101.

Lucas, Jr., Robert E. On the Mechanics of Economic Development. En: Journal of Monetary Economics. No. 22, (1988): 3-42.

McEwan, Patrick J. The indigenous test score gap in Bolivia and Chile. En: Economic Development and Cultural Change, Vol. 54. No. 1 (2004):157-90, http://academics.wellesley.edu/Economics/mcewan/PDF/ indigenous.pdf.

Mina Calvo, Alejandro. Factores asociados al logro educativo a nivel municipal. Bogotá: Universidad de Los Andes, 2004 (Tesis de Maestría)

Muñoz-Repiso, Mercedes y F. Javier Murillo. Un balance provisional sobre la calidad de la educación. Eficacia escolar y mejora de la escuela. En: Organización y Gestión Educativa. Vol. 9, No. 4 (2004). [En: REICE. Revista Iberoamericana sobre Calidad, Eficacia y Cambio en Educación, http://www.rinace.net/ reice/numeros/arts / vol8num2/art10.pdf: 177-186].

Organisation for Economic Co-operation and Development (OECD). Are Students Ready for a Technology-Rich World? What PISA studies tell us. París: OECD, 2006, http://www.oecd.org/edu/school/programmefor nter nationalstudentassessmentpisa/35995145.pdf.

Organisation for Economic Co-operation and Development (OECD). Knowledge and skills for life: first results from PISA 2000. París: OCDE, 2001, http://www.oecd.org/education/school/programmeforinternatio nal studentassessmentpisa/33691620.pdf.

Piñeros Jiménez, Luis Jaime y Alberto Rodríguez Pinzón. Los insumos escolares en la educación secundaria y su efecto sobre el rendimiento académico de los estudiantes: Un estudio en Colombia. Washington, D.C.: The World Bank/Latin America and the Caribbean Region/Department of Human Development, 1998.

Rebelo, Sergio. Long-Run policy analysis and long-run growth. En: The Journal of Political Economy. Vol. 99, No. 3 (jun., 1991): 500-521. 
Romer, Paul M. Increasing Returns and Long-Run Growth. En: The Journal of Political Economy. Vol. 94, No. 5 (oct., 1986): 1002-1037.

Sanders, W., y Rivers, J. Cumulative and Residual Effects of Teachers on Future Student Academic Achievement. Knoxville, TN: University of Tennessee/Value-Added Research and Assessment Center, 1996, http://www. cgp.upenn.edu/pdf/Sanders_Rivers-TVASS_teacher\%20effects.pdf.

Stein, Ernesto H.; Mariano Tommasi, Koldo Echebarría, Eduardo Lora y J. Mark Payne. The politics of policies: economic and social progress in Latin America. Washington, DC: Banco Interamericano de Desarrollo, 2006.

Vaillant, Denise. Las políticas TIC en los sistemas educativos de América Latina: Caso Uruguay. Buenos Aires: Fondo de las Naciones Unidas para la Infancia (UNICEF), 2013.

Vegas, Emiliana y Jenny Petrow. Incrementar el aprendizaje estudiantil en América Latina. Washington D.C.: Banco Mundial, 2008.

Willms, J. Douglas y Marie-Andree Somers. Family, Classroom, and School Effects on Children's Educational Outcomes in Latin America. En: School Effectiveness and School Improvement. Vol. 12, No. 4, (dic., 2001): 409-445.

Woessmann, Ludger y Thomas Fuchs. Families, schools, and primary-school learning: evidence for Argentina and Colombia in an international perspective. Washington, DC: Banco Mundial, 2005 (Documento de trabajo 3537).

Wößmann, Ludger. Schooling resources, educational institutions and student performance: the international evidence". Oxford Bulletin of Economics and Statistics. Vol. 65. No. 2, (may., 2003): 117-170.

Wolff, Laurence; Juan Carlos Navarro y Pablo González (eds.). Private education and public policy in Latin America. Washington, DC: Partnership for Educational Revitalization in the Americas (PREAL), 2003. 